\title{
$\angle S$ Research Square \\ Incidence and risk factors for postictal delirium in patients after electroconvulsive therapy in China
}

\section{Nan Cheng}

Third Affiliated Hospital of Sun Yat-Sen University

Dezhao Liu

Third Affiliated Hospital of Sun Yat-Sen University

\section{Yue Guo}

Third Affiliated Hospital of Sun Yat-Sen University

Jingyi Du

Third Affiliated Hospital of Sun Yat-Sen University

\section{Ping Xiang}

Southern Medical University Nanfang Hospital

\section{Ziqing Hei}

Third Affiliated Hospital of Sun Yat-Sen University

Xiang Li ( $\square$ lixiang27@mail.sysu.edu.cn )

Third Affiliated Hospital of Sun Yat-Sen University https://orcid.org/0000-0003-4997-9339

\section{Research article}

Keywords: cognition, delirium, electroconvulsive therapy, incidence, risk factors

Posted Date: June 10th, 2020

DOI: https://doi.org/10.21203/rs.3.rs-32553/v1

License: () (1) This work is licensed under a Creative Commons Attribution 4.0 International License.

Read Full License 


\section{Abstract}

Aim: This study aimed to elucidate the incidence and risk factors for postictal delirium (PID) among Chinese patients undergoing electroconvulsive therapy (ECT).

Methods: In this retrospective study, 203 patients who underwent ECT in the Third Affiliated Hospital of Sun Yat-Sen University from July 2016 to July 2017 had their PID severity measured by a previously developed scale. For data analysis, two groups were created: PID patients and non-PID patients. The groups were analyzed based on three types of independent variables: patient-related, electroconvulsive treatment, and hemodynamic variables. Data analysis was performed through descriptive statistics, Chisquared tests, Fisher exact tests, and/or independent sampled t-tests. Logistic regression analysis was used to identify independent risk factors for postictal delirium $(P<0.05)$.

Results: Results showed that 81 patients (39.9\%) developed moderate to severe PID in their first ECT session. Patients receiving ECT for the first time $(P=0.016)$, agitation before the ECT $(P=0.028)$, and high heart rate variances $(P=0.044)$ were identified as risk factors for PID, and they were significantly correlated with the occurrence of moderate to severe PID $(P<0.01)$.

Conclusion: The patients receiving ECT for the first time, with agitation states before ECT and/or with high heart rate variance during ECT procedures might be at higher risk for moderate to severe PID. The medical staff related to this type of treatment may benefit from detailed knowledge about the aforementioned risk factors for predicting PID and to anticipate the best possible management for these patients.

\section{Introduction}

It has been more than 80 years since the introduction of electroconvulsive therapy (ECT) into modern psychiatry, and ECT has become an effective treatment for mental disorders, particularly for treatmentresistant cases and seriously challenging situations. ${ }^{1-3}$ Numerous analyses have approved the effect of ECT and considered it remarkable for those who have been unresponsive to or who tolerate the conventional treatments such as pharmacotherapy and psychotherapy. ${ }^{1}$ However, some adverse effects still exist after ECT procedures, which might be a significant concern.

One such adverse effect is postictal delirium (PID), which has been identified as a relatively common adverse effect that usually occurs immediately after the ECT procedure, with a reported incidence for at least one third of the treatments. ${ }^{4,5}$ The clinical manifestations of the PID state mainly include lack of awareness, perceptual disturbance, agitation, and sometimes erratic and/or violent behavior. A series of adverse outcomes, such as falls, fractures, need for extra nursing care, and physical restraint related to psychomotor agitation might come as a result of PID. ${ }^{3-5}$ Thus, a more thorough and detailed recognition of the risk factors related to PID could be helpful in preventing and treating this unwanted outcome that is commonly associated with ECT. 
However, the pathogenesis of PID remains uncertain and, currently, only a few of the proposed preventive and/or therapeutic measures have presented conclusive outcomes. Previous research has regarded that the etiology of PID might be multifactorial, including an interrelationship between predisposing and precipitating factors. ${ }^{3}$ To date, some factors have been reported to be associated with an increased risk for the PID state, such as catatonic features, cerebrovascular disease, dementia, electrode placement, high intensity stimuli, and prolonged seizures. ${ }^{3}$ However, to the best of our knowledge, there has been few studies regarding the incidence and risk factors of PID in China. Furthermore, few studies demonstrated whether the hemodynamic variables of the patients during ECT were related to the occurrence of PID.

Therefore, we conducted the present analysis to determine the incidence and risk factors for PID among Chinese patients who underwent ECT while also analyzing their hemodynamic variables.

\section{Methods}

\section{Patients}

This study was approved by the Research Ethics Committee of the Third Affiliated Hospital of Sun YatSen University. Due to the retrospective and anonymous nature of the current study, written informed consent was waived by the research ethics committee. We included all the patients who received ECT at the Third Affiliated Hospital of Sun Yat-Sen University, between July 2016 and July 2017. Clinical data were obtained retrospectively from available hospital records. Among the collected data, only the patients' first ECT treatment (of a series) was analyzed. The exclusion criteria were: patients with cognitive impairment before treatment, clinically significant abnormal findings in laboratory test or electrocardiography, epilepsy, alcoholism or drug abuse, clinically significant organic or neurological disease, and patients with incomplete hospital records.

\section{Procedures}

After the patient's arrival to the operating room (OR), the anesthetist would make an assessment for the sedation level through the Richmond Agitation-Sedation Scale (RASS). The RASS is a 10-point sedation scale ranging from -5 to 4 . Negative scores represent increasing levels of sedation, with -5 corresponding to an unarousable patient, whereas positive scores represent increasing levels of agitation with 4 corresponding to a combative patient. ${ }^{6}$ According to the analysis of Reti et al., a patient with a RASS score $\geq 2$ was considered hazardous because a RASS score of 2 was the threshold for dangerous levels of agitation. ${ }^{4}$

Before ECT, routine monitoring of electrocardiogram (ECG), pulse oxygen saturation $\left(\mathrm{SpO}_{2}\right)$ and blood pressure (BP) were performed for each patient. Anesthesia was induced by $1.5 \mathrm{mg} / \mathrm{kg}$ of propofol, and then $0.7 \mathrm{mg} / \mathrm{kg}$ succinylcholine was intravenously injected to act as a muscle relaxant. The ECT procedure was performed by bitemporal electrode stimulation with the Thymatron IV System (Somatics Inc., Lake Bluf, IL, USA). To determine the initial stimulus dose for the first ECT session, the patients' age 
half-value was analyzed. For the following ECT sessions, the stimulus dose was adjusted based on the seizure quality throughout the whole ECT session, and it was common practice to enhance the stimulus dose about 1.5 times for each subsequent session. We have evaluated seizure quality in accordance with previous studies that determined the seizure morphology and length should be taken into account in this process. $^{7}$

For each treatment, the heart rate (HR) and mean arterial pressure (MAP) were recorded at baseline, 0,1 , 3, 5 and 10 min after the electrical stimulation ended. Moreover, the post-ECT adverse effects - including agitation, headache and nausea - were also measured.

\section{Assessment of PID}

The patients' PID level was evaluated according to the delirium scoring scale described by Kikuchi et al. ${ }^{5}$ The scale ranged from 0 (no delirium) to 3 (severe delirium) and was scored by the researchers. For further details related to this scale, refer to Table 1.

\section{Independent variables}

The independent variables assessed for each patient included patient-related variables (age, weight, gender, diagnosis, duration of psychiatric disorder and RASS score), ECT treatment variables (seizure length) and hemodynamic variables (heart rate and mean arterial pressure at baseline and immediately after ECT). Moreover, the variances of patients' hemodynamic variables were calculated according to the formula presented in a study by Hirsch: variance = where is a patient's HR or MAP at a particular time point $x$, is the mean of the patient's HR or MAP, and $n$ is the number of HR and MAP measurements. ${ }^{8}$ In this study, represents HR or MAP at baseline and immediately after the electrical stimulation; represents the mean value of HR or MAP at the aforementioned two time points; $n$ represents two measurements.

\section{Statistical analysis}

Based on the study from Kikuchi et al., a PID score of $\geq 2$ was regarded as a clinical problem. ${ }^{5}$ Thus, the collected variables were compared between two groups: the non-PID group, including patients with scores of $0-1$ (no to mild delirium); and the PID group, including patients with scores of 2-3 (moderate to severe delirium). Continuous variables were expressed by means and standard deviation, or medians and interquartile ranges, whereas the categorical data were expressed by their percentages. Bivariate relationships between these variables were examined using Chi-squared tests, Fisher exact tests, or independent sampled t-tests. Subsequently, the logistic regression model was built to determine independent risk factors of PID, and was performed with all the significantly different factors found in the univariate comparison process between the PID and non-PID groups. A value of $P<0.05$ was considered significant.

\section{Results}


According to the inclusion and exclusion criteria, a total of 203 patients were analyzed. Figure 1 shows how many patients were excluded by each of these categories.

\section{Incidence of PID}

Among the patients, 51 individuals had no delirium (score 0); 71 individuals had mild delirium (score 1); 67 individuals had moderate delirium (score 2); and 14 individuals had severe delirium (score 3). As a delirium score of $\geq 2$ was considered a clinical problem, ${ }^{5}$ the incidence of PID (score $2-3$ ) was $39.9 \%$ $(81 / 203)$. Figure 1 shows number of participants, exclusion criteria and incidence rate.

\section{Analysis of risk factors for PID}

Patients with delirium scores $\geq 2$ were assigned to the PID group, and the patients with delirium scores < 2 delirium were assigned to the non-PID group. Multiple comparisons were performed on the various factors between the PID and non-PID groups, and significant differences were observed in rate of patients receiving ECT for the first time $(P=0.016)$, rate of RASS score $\geq 2(P=0.028)$ and HR variance $(P=$ 0.044 ) between the two groups (Table 2 ).

\section{Logistic regression analysis}

The multivariate analysis identified receiving ECT for the first time $(P=0.003)$, RASS score $\geq 2$ before ECT $(P=0.003)$ and HR variance $(P=0.004)$ as significant and independent risk factors for PID (Table 3$)$.

\section{Discussion}

In the present study, we revealed the incidence of PID to be $39.9 \%$ for Chinese patients undergoing ECT, a value supported by the previous research of Kikuchi et al., in which they utilized the same delirium measure and identified the incidence of PID to be $36 \%{ }^{5}$

Furthermore, receiving ECT for the first time, RASS score $\geq 2$ before ECT and high HR variance were identified as significant and independent risk factors for PID. During the ECT procedure, hyperdynamic responses are usually evoked by parasympathetic discharge that coincides with electrical stimulation, which might result in a $30 \%$ to $40 \%$ increase in blood pressure, and an increase over $20 \%$ in $\mathrm{HR} .{ }^{9,10}$ In this analysis, we did not find significant differences either on MAP or on HR values immediately after the ECT between the two patient groups. Interestingly, when comparing HR fluctuation, we found a larger variance in those with PID. This finding is in line with previous suggestions that the increase in the variable hemodynamic fluctuation, not the absolute or relative hemodynamic value, might be a predictor of PID. ${ }^{8,11}$ Nevertheless, it is still unclear as to why increased HR fluctuation causes an increased chance of PID. In this regard, Jooyoung et al. speculated that higher HR fluctuations could lead to an instability in the autonomic nervous system, which may possibly be associated with delirium. ${ }^{11}$ Consequently, it can be inferred that a higher level of control over patients' HR, as well as over their blood pressure, during ECT may help prevent PID. 
We deem important to note that PID was more frequently observed in patients that had higher RASS scores before ECT, which may lead to an understanding that more agitated patients experience PID more frequently. The impact of agitation before ECT on PID has also been evaluated by the study from Gomez and Dally: they found that the incidence and severity of post treatment agitation was significantly greater when ECT was performed without prior patient tranquillization. ${ }^{12}$ However, the treatment for agitation before ECT remains controversial, as some conventional medication administered to prevent patients' agitation, such as benzodiazepines, were shown to interfere with seizure duration and lead to numerous, sometimes serious, adverse effects. ${ }^{13,14}$ Although the treatment for agitation before ECT is inconclusive, caution should be exercised when considering patients with previous history of anxiety to undergo ECT, as they may be more propense to develop PID.

Although some demographic characteristics, such as age, gender and diagnosis, were considered as risk factors for PID in many medical settings, our results showed that differences in these characteristics did not seem to contribute to the appearance of PID after ECT. ${ }^{4,5}$ Further, we did not find any association between PID, weight and duration of illness; notwithstanding, when referring to how many times patients had received ECT, we found that patients who were undergoing this procedure for the first time were more propense to develop PID. Additionally, we did not find any correlation that indicated that seizure length predicted the occurrence of PID, which was inconsistent with the research by Reti et al. ${ }^{4}$ It is worth noting that the seizure length and stimulation dose found in Reti's research seemed to be greater than those utilized in our study. Therefore, there is a possibility that the relatively short seizure length we have provided in this study was not adequate to reveal how seizure length may affect PID.

In this study, all patients were adequately anesthetized with propofol and succinylcholine before ECT. We did not find an association between the dose of anesthetics and PID, which was consistent with the findings of previous studies. ${ }^{4,15}$ Further, previous research showed that when ECT is administered with no anesthetic agent, PID occurs more frequently and tend to present more serious symptomatic outcomes. ${ }^{16}$ Thus, it is reasonable to presume that adequate anesthesia during the ECT procedure acts as a protective factor for PID, and that it may decrease its effects.

Our study has several limitations. Firstly, collected data referred only to the first ECT administration, and is common knowledge in the field that, if the patient experiences PID symptoms in the first procedure, subsequent applications undergo modifications to better suit each patients' needs. Thus, it is not known whether these found risk factors would relate to PID in subsequent ECT treatments, and future studies should seek to resolve this conundrum.

Secondly, the scale utilized in this study to measure PID has yet to be more widely used. Therefore, the knowledge about the specificity and sensitivity of this scale measurement was still limited the time of this study. Future studies are warranted to define the best suited approaches for PID measurement, and if our chosen measure is indeed accurate and valid for this type of measurement.

\section{Conclusion}


In this study, results showed that the incidence of PID among Chinese patients was 39.9\%. Moreover, receiving ECT for the first time, agitation before ECT and higher HR variance were identified as significant and independent risk factors for PID. These findings suggest that the medical staff related to ECT treatment may benefit from detailed knowledge about the aforementioned risk factors for predicting PID and to anticipate the best possible management for these patients.

\section{Declarations}

\section{Acknowledgments}

The authors thank the patients and their families for participating in this study. We also thank Dr. Chun Hao from School of Public Health, Sun Yat-sen University for her assistance with statistical analysis. This study was supported by the Sun Yat-sen University Clinical Research 5010 Program (2017016).

\section{Competing interests}

The authors declare no conflict of interest.

\section{Author contributions}

Conceived and designed the experiments: Z.H. and X.L. Collected the Clinical data: N.C., D.L and M.Z. Analyzed and interpreted the data: P. X. and M.Z. Wrote the first draft of the manuscript, and handled subsequent drafts after receiving coauthors' feedback: J.D., Y.G. and X.L. All of the authors contributed to the final version of the paper.

\section{Funding}

This study was supported by the Fundamental Research Funds for the Central Universities (20ykpy25) and Sun Yat-sen University Clinical Research 5010 Program (2017016).

\section{References}

1. Group UER. Efficacy and safety of electroconvulsive therapy in depressive disorders: a systematic review and meta-analysis. Lancet 2003; 361: 799-808.

2. The Lancet P. Getting better. The lancet Psychiatry 2018; 5: 287.

3. Tsujii T, Uchida T, Suzuki T, Mimura M, Hirano J, Uchida H. Factors Associated With Delirium Following Electroconvulsive Therapy: A Systematic Review. The journal of ECT 2019.

4. Reti IM, Krishnan A, Podlisky A, Sharp A, Walker M, Neufeld KJ, Hayat MJ. Predictors of electroconvulsive therapy postictal delirium. Psychosomatics 2014; 55: 272-9.

5. Kikuchi A, Yasui-Furukori N, Fujii A, Katagai H, Kaneko S. Identification of predictors of post-ictal delirium after electroconvulsive therapy. Psychiatry and clinical neurosciences 2009; 63: 180-5. 
6. Ely EW, Truman B, Shintani A, Thomason JW, Wheeler AP, Gordon S, Francis J, Speroff T, Gautam S, Margolin R, Sessler CN, Dittus RS, Bernard GR. Monitoring sedation status over time in ICU patients: reliability and validity of the Richmond Agitation-Sedation Scale (RASS). Jama 2003; 289: 2983-91.

7. Kranaster L, Hoyer C, Janke C, Sartorius A. Bispectral index monitoring and seizure quality optimization in electroconvulsive therapy. Pharmacopsychiatry 2013; 46: 147-50.

8. Hirsch J, DePalma G, Tsai TT, Sands LP, Leung JM. Impact of intraoperative hypotension and blood pressure fluctuations on early postoperative delirium after non-cardiac surgery. British journal of anaesthesia 2015; 115: 418-26.

9. Li X, Tan F, Jian CJ, Guo N, Zhong ZY, Hei ZQ, Zhou SL. Effects of small-dose dexmedetomidine on hyperdynamic responses to electroconvulsive therapy. Journal of the Chinese Medical Association : JCMA 2017; 80: 476-81.

10. Ding Z, White PF. Anesthesia for electroconvulsive therapy. Anesthesia and analgesia 2002; 94: 135164.

11. Jooyoung O, Dongrae C, Jongin K, Jaeseok H, Jaesub P, Se Hee N, Cheung Soo S, Jae-Jin K, Jin Young P, Boreom L. Changes in heart rate variability of patients with delirium in intensive care unit. Conference proceedings : Annual International Conference of the IEEE Engineering in Medicine and Biology Society IEEE Engineering in Medicine and Biology Society Annual Conference 2017; 2017: 3118-21.

12. Gomez J, Dally P. Intravenous tranquillization with ECT. The British journal of psychiatry : the journal of mental science 1975; 127: 604-8.

13. Manchester KR, Lomas EC, Waters L, Dempsey FC, Maskell PD. The emergence of new psychoactive substance (NPS) benzodiazepines: A review. Drug testing and analysis 2018; 10: 37-53.

14. Oosthuizen P, Emsley R, Jadri Turner H, Keyter N. A randomized, controlled comparison of the efficacy and tolerability of low and high doses of haloperidol in the treatment of first-episode psychosis. The international journal of neuropsychopharmacology 2004; 7: 125-31.

15. Devanand DP, Briscoe KM, Sackeim HA. Clinical Features and Predictors of Postictal Excitement. Convulsive therapy 1989; 5: 140-46.

16. Gallegos J, Vaidya P, D'Agati D, Jayaram G, Nguyen T, Tripathi A, Trivedi JK, Reti IM. Decreasing adverse outcomes of unmodified electroconvulsive therapy: suggestions and possibilities. The journal of ECT 2012; 28: 77-81.

\section{Tables}

Table 1. Scoring system for postictal delirium assessment ${ }^{5}$ 
Level of delirium

severity

No

delirium

Mild

delirium

Moderate

delirium

Severe

delirium
Description

Delirium

score

/

0

(n)

Possible to cope with the delirium with a verbal command alone

1

Necessary to manually restrain the patient who exhibits excitement and

2 thrashing

Delirium prolonged for two to three minutes and manual restraint of the

3 patient necessary three times, injection of a sedative drug required to end the delirium 
Table 2. Demographic and clinical characteristics of patients $(n=203)$

Variable No or mild PID Moderate-severe PID $(n=81) \quad P$ value $(n=122)$

Age (years)

Weight $(\mathrm{kg})$

Gender (M/F)

Duration of illness (years)

Number of ECT sessions

Receiving ECT for the first time ( $\mathrm{N}[\%])$

RASS score $\geq 2$ before ECT

Diagnosis

Major depressive disorder (N [\%])

Bipolar disorder ( $\mathrm{N}[\%])$

Schizoaffective disorder ( $[\%])$

ECT treatment variables

seizure length (seconds)

\section{Hemodynamic variables}

Base HR

Peak HR

$\mathrm{HR}$ variance $\left(\mathrm{bpm}^{2}\right)$

Base MAP $(\mathrm{mmHg})$

Peak MAP $(\mathrm{mmHg})$

MAP fluctuation $\left(\mathrm{mmHg}^{2}\right)$

$28.7 \pm 9.8$

$60.2 \pm 10.5$

$47 / 75$

$3.7 \pm 3.4$

$8.0 \pm 0.8$

$86 / 122(70.5 \%)$

10/122

73

36

13

$35.1 \pm 7.6$

$36.3 \pm 9.9$

0.356

$29.1 \pm 10.9$

0.813

$58.7 \pm 8.4$

0.286

$31 / 50$

0.971

$3.9 \pm 3.3$

0.746

0.302

$7.9 \pm 0.7$

0.016

69/81(85.2\%)

0.028

0.731

51

24

6

$15 / 81$

51

(




\begin{tabular}{|llllll|}
\hline Variable & $\begin{array}{l}\text { Regression } \\
\text { Coefficient }\end{array}$ & Odds Ratio & \multicolumn{2}{c}{$95 \%$ Confidence Interval } & Pvalue \\
\cline { 4 - 5 } & & Lower & \multicolumn{2}{c|}{ Upper } & \\
Receiving ECT time & -1.229 & 0.293 & 0.131 & 0.654 & 0.003 \\
\hline RASS score & 0.944 & 2.570 & 1.387 & 4.762 & 0.003 \\
\hline HR variance & 0.002 & 1.002 & 1.000 & 1.003 & 0.004 \\
\hline
\end{tabular}

PID, Post-ictal Delirium; ECT, Electroconvulsive Therapy; HR, Heart Rate.

\section{Figures}

\section{Initial hospital records}

Patients receiving electroconvulsive therapy treatments at the Third Affiliated Hospital of Sun Yat-sen University, between July 2016 and July $2017(n=268)$.

A total of 203 patients were included, with the delirium incidence of $39.9 \%$ in the first treatment sessions.

\section{Figure 1}

Flow chart of patients' inclusion and exclusion 